\title{
Effect of exercise on diastasis recti abdominis among the primiparous women: a quasi-experimental study
}

\author{
Mahalakshmi V. ${ }^{1}$, Sumathi G. ${ }^{2}$, Chitra TV $^{3}$, Ramamoorthy V. $^{2}$
}

\author{
${ }^{1}$ PSG College of Physiotherapy, Coimbatore, Tamil Nadu, India \\ ${ }^{2}$ Physical Medicine and Rehabilitation, PSG Hospitals, Coimbatore, Tamil Nadu, India \\ ${ }^{3}$ Department of Obstetrics and Gynaecology, PSG Hospitals, Coimbatore, Tamil Nadu, India
}

Received: 11 October 2016

Revised: 08 November 2016

Accepted: 09 November 2016

\author{
*Correspondence: \\ Mahalakshmi V., \\ E-mail: mahas_v@yahoo.com
}

Copyright: () the author(s), publisher and licensee Medip Academy. This is an open-access article distributed under the terms of the Creative Commons Attribution Non-Commercial License, which permits unrestricted non-commercial use, distribution, and reproduction in any medium, provided the original work is properly cited.

\begin{abstract}
Background: Diastatis recti abdominis (DRA) occurs most commonly in pregnant women owing to weakness of the abdominal musculature resulting from maternal hormone influences and increased stretch of the rectus abdominis induced by the expanding uterus. As there are musculoskeletal complications following DRA, there is a need to implement DRA corrective exercises in preventing or treating DRA following child birth. The objective of the study was to investigate the effects of Diastasis rectus abdominis corrective exercises in reducing DRA among the primiparous women.

Methods: Primiparous women who had DRA of more than 2 finger width at their umbilical level or a bulging rectus abdominis on contraction following a vaginal delivery (VD) or cesarean section (LSCS) were included as per the inclusion and exclusion criteria.

Results: Fifty four and forty two women in the VD and LSCS group respectively participated in the study. Among them only 12 and 9 women from each group respectively performed the DRA corrective exercises for 6 weeks. There was a significant improvement in the reduction of DRA within the groups at $p<0.01$. Comparison of reduction in DRA between VD and LSCS did not show any significant difference at $p>0.05$ which indicated that both the groups showed the same level of improvement.

Conclusions: DRA corrective exercises performed by the primiparous women with DRA in their postpartum period immediately following ND or a month after the LSCS for 6 weeks were found to be effective in reducing DRA.
\end{abstract}

Keywords: Diastasis recti, Finger width, Post natal, Transverse abdominis strengthening

\section{INTRODUCTION}

Diastasis recti abdominis (DRA) is a midline separation of the rectus abdominis (RA) muscles at the linea alba in the abdominal wall. A palpable midline gap of more than $2.5 \mathrm{~cm}$ or any visible bulging on exertion is clinically considered as DRA. Among patients with urogynecological disorders, $52 \%$ were found to have a DRA and $66 \%$ had at least one type of pelvic floor dysfunction (stress urinary incontinence, fecal incontinence and/or pelvic organ prolapse)., ${ }^{1,2}$ DRA occurs most commonly in pregnant women owing to weakness of the abdominal musculature resulting from maternal hormone influences and increased stretch of the RA induced by the expanding uterus. DRA can occur in varying degrees during pregnancy and may not resolve spontaneously in the postpartum period. ${ }^{1}$

Weakness in the abdominal wall associated with DRA interferes with the ability of the abdominal muscles to stabilize the trunk, which in turn can lead to poor posture and umbilical hernia., ${ }^{3,4,9}$ 
Moreover, weakness in the core muscles contributes to insufficient force closure of the sacroiliac joint leading to pelvic instability. This can eventually lead to low back and hip pain. ${ }^{1}$

Facilitation, concentric activation, and stabilization of the abdominal muscles occur during pelvic tilting with abdominal bracing and pelvic clock exercises used to correct diastasis recti. ${ }^{3}$ Many studies have been performed on DRA correction exercises for the postnatal women following vaginal delivery. ${ }^{1,6,7}$ On the other hand, only few studies have dealt with DRA correction in women who had a cesarean section. Despite of the type of delivery (vaginal or cesarean), postnatal mothers do not show much interest in performing exercises immediately following delivery. They seek medical advice only when they experience low back pain or pelvic floor dysfunction in future. Following re-education of the transversus abdominis (TA), Sheppard documented successful reduction of DRA and a decrease in postnatal back pain. The importance of re-education of the deep stabilizing muscles of the trunk, including the TA, has been well documented. ${ }^{5}$ Coldron et al. measured the inter-recti distance from day 1 to 1 year postpartum and stated that characteristics of RA thickness and width, and the inter recti distance (IRD) did not revert back to normal state by the end of one year postpartum. Persistent increased IRD may cause decreased stiffness of the anterior abdominal wall and predispose to a mechanical disadvantage. Exercises targeted at achieving normal RA width, thickness, and length and correction of IRD without loading and compressing the lumbar spine are required. ${ }^{8}$ Hence, there is a need to implement DRA corrective exercises in preventing or treating DRA following child birth. The aim of the current study was to investigate the effects of DRA corrective exercises in reducing DRA among the primiparous women who underwent vaginal delivery (VD) (including forceps and vacuum assisted vaginal delivery [VAVD]) or Lower segment cesarean section (LSCS).

\section{METHODS}

A quasi-experimental study design was used. The study was conducted at the Physiotherapy Department of PSG Hospital, Tamil Nadu, India. Ethical clearance was obtained from the Institutional Human Ethics Committee of PSG Institute of Medical Sciences and Research. Prior to data collection, a signed informed consent was obtained from all participants.

Participants were recruited by word of mouth between August 2014 and May 2015 from PSG Hospitals. Primiparous women, aged between 19 and 42 years, who had DRA of more than 2 finger width at their umbilical level or a bulging of RA on contraction following a vaginal delivery or cesarean section were included if they were free from any other postnatal complications. Those women who were performing abdominal strengthening exercises during pregnancy were excluded as these exercises may have an influence on the occurrence/characteristics of DRA. Moreover, there is an association of DRA during pregnancy with lack of regular exercise during pregnancy. ${ }^{18}$

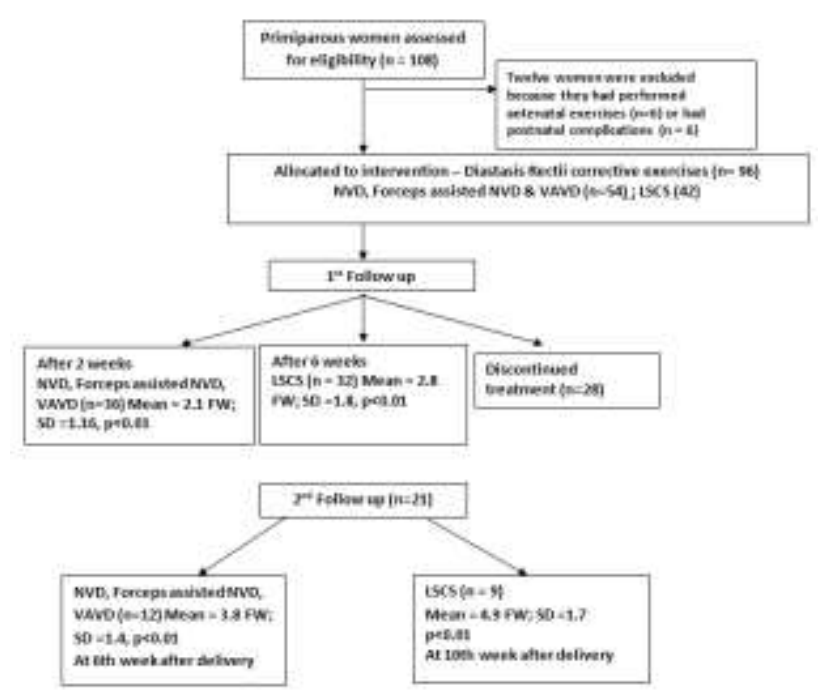

Figure 1: Flow chart showing study design and findings.

Awareness about DRA during pregnancy and its effect on the abdominal muscle strength, lumbo-pelvic stability, back pain and umbilical hernia was given to all the participants, through information pamphlets, on their 3rd postnatal or 4th postoperative day. The study included a protocol of TA strengthening exercises along with pelvic floor muscles exercises to be performed immediately following the vaginal delivery and a month after the cesarean section (Table 1). At the initial visit, the patients received education on correct body mechanics, posture, and appropriate exercises to approximate the RA muscle without increasing intra-abdominal pressure. Selfchecking of DRA was taught to all participants. The corrective exercises were taught on the 3rd postnatal day for women who had a vaginal delivery and they were reviewed after 2 weeks and 6 weeks from the date of delivery. Postnatal women with a cesarean section were taught the corrective exercises at the 2nd week and reviewed at 6th and 10th week following delivery. DRA was reassessed on their subsequent visits to analyze the changes in DRA. Abdominal exercises were postponed until about 4 weeks after cesarean birth as it may interfere with the healing process and also may increase the incision pain. ${ }^{11,12}$ All postnatal women who had improvement in DRA at the end of 6 weeks were taught the abdominal strengthening exercises involving RA, external and internal oblique muscles. Special precautions such as splinting the abdominal muscles or approximating them during coughing or sneezing, avoiding lifting of heavy weights, bending down with knees (squatting), avoiding unsuitable abdominal exercises like sit ups, crunches, trunk twisting movements or straight leg raises were also taught to all the participants. 


\section{Statistical analysis}

With SPSS version 19, a comparison of the baseline and follow-up (DRA) values was performed using paired $t$ test. A $p$ value $\leq 0.05$ was used as the level of statistical significance. The effect size (d) was determined using the formula: Mean change score/ standard deviation of preintervention score where mean change score is the difference between mean values of pre- and postintervention scores. In this study, Cohen's classification was used to interpret the effect sizes as small $(d=0.2)$, medium $(\mathrm{d}=0.5)$ or large $(\mathrm{d} \geq 0.8) .^{13}$

\section{Treatment protocol}

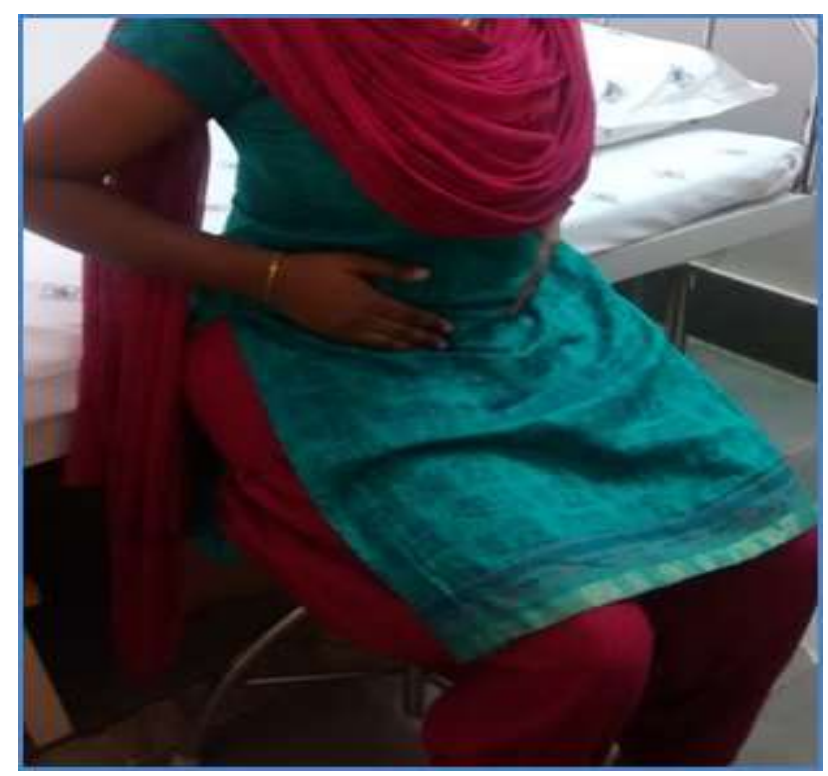

Figure 2: Seated squeeze and seated transverse.

\section{Seated squeeze}

$\checkmark$ Patient is asked to sit on a bench /chair and place both hands on the abdomen one inch inferior and medial to ASIS to feel for the transverse abdominis muscle contraction

$\checkmark$ Patient should draw in the navel towards spine without holding her breath and feel for TA contraction

$\checkmark \quad$ The movement is very small

$\checkmark$ The contraction is maintained for 30 counts

$\checkmark$ Repeated 10 times

$\checkmark \quad$ Can be done frequently

\section{Seated transverse}

$\checkmark \quad$ Same as the previous exercise but need to imagine the abdomen as levels of floor, with navel as $1^{\text {st }}$ floor, and contract the muscle maximally up to $5^{\text {th }}$ floor with imagination

$\checkmark$ TA Contraction is started from $1^{\text {st }}$ to $3^{\text {rd }}$ floor of spine $\checkmark$ Then TA contraction to be performed continuously for 100 repetitions from $3^{\text {rd }}$ to $5^{\text {th }}$ floor

$\checkmark \quad 5$ sets per day

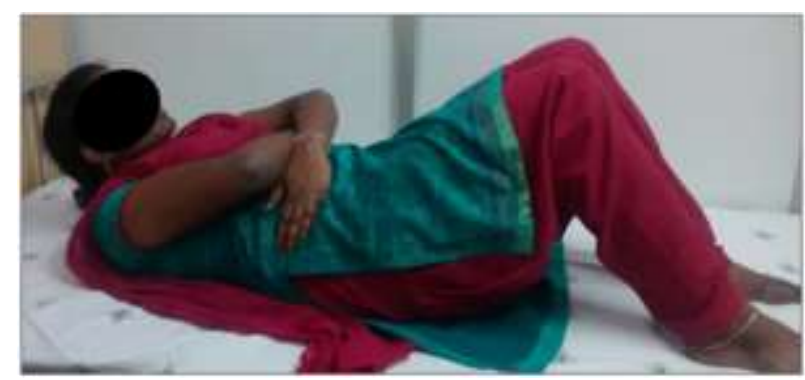

Figure 3: Approximation exercise.

\section{Approximation exercises}

$\checkmark \quad$ Lying on the back with knees bent without a pillow underneath, patient should cross her arms and place each hand on either side of her abdomen

$\checkmark$ Hands should be held across the abdomen and squeeze the muscles together

$\checkmark$ Patient should breathe in and when she breathes out she should raise her head and hold for 10 seconds

$\checkmark \quad$ Slowly she should lower her head and shoulders back to the mat/bed and relax her hands

$\checkmark$ Perform 25 repetitions twice daily

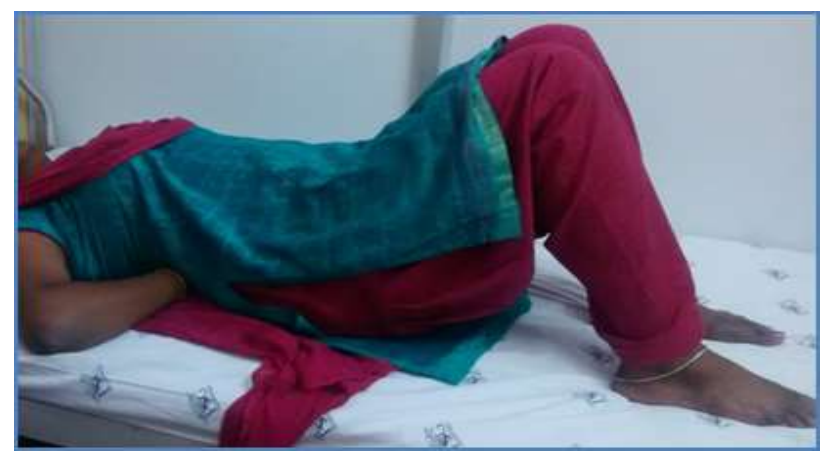

Figure 4: Pelvic tilt.

\section{Pelvic tilt}

$\checkmark \quad$ Lying on the back,patient should tilt her pelvis posteriorly as if flattening the lumbar lordosis and hold the position for 10 seconds

$\checkmark \quad$ Should perform 25 repetitions; twice daily

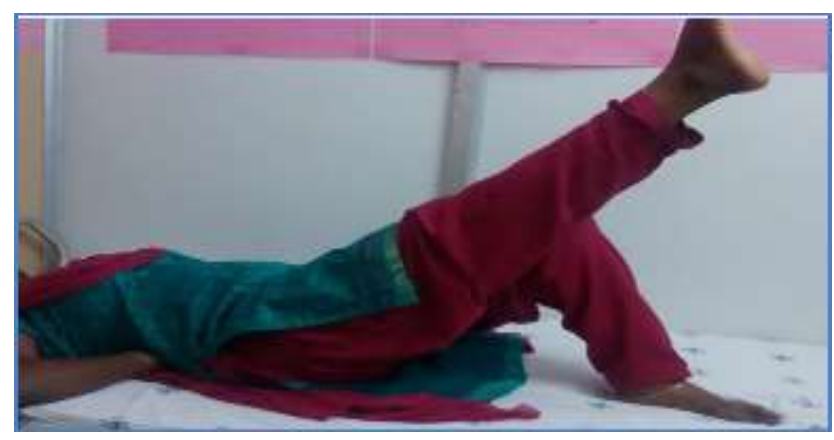

Figure 5: Heel drop with core activation. 


\section{Heel drop with core activation}

$\checkmark$ Engaging the core and pelvic floor muscles, patient should lift one leg up and slowly bring it down while maintaining the contraction of these muscles and then repeat the exercise with the other leg

$\checkmark$ Should perform 25 repetitions, twice daily

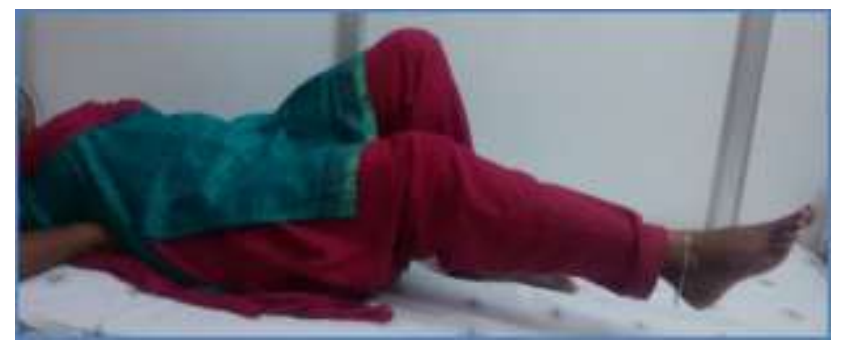

Figure 6: Heel slide with core activation.

\section{Heel slide with core activation}

$\checkmark \quad$ Starting with both knees bent and feet flat on the bed:

$\checkmark$ Patient should engage the core and pelvic floor muscles

$\checkmark \quad$ The patient should slide one heel along the bed and flex to the starting position by maintaining the contraction of the transverse abdominis and pelvic floor muscles. The exercise has to be performed on alternate legs

$\checkmark \quad$ Should perform 25 repetitions, twice daily

\section{RESULTS}

One Hundred and eight primiparous women with VD or LSCS volunteered for the study. Among them, six women who performed their antenatal exercise programme and six women with postnatal complications were excluded for the study. Therefore, 96 women, 54 with VD and 42 with LSCS, with a mean age of $26.25( \pm$ 3.61) years were included for the study.

Table 1: Reduction of DRA among NVD and LSCS Primiparous women during first follow up.

\begin{tabular}{|c|c|c|c|c|c|}
\hline \multirow{2}{*}{$\begin{array}{l}\text { Type of } \\
\text { delivery }\end{array}$} & \multirow{2}{*}{$\begin{array}{l}\text { No. of } \\
\text { participants }\end{array}$} & \multirow{2}{*}{$\begin{array}{l}\text { Mean age } \\
\text { (years) }\end{array}$} & \multicolumn{2}{|c|}{ Mean and SD of DRA } & \multirow[t]{2}{*}{ Paired $\mathrm{t}$ test } \\
\hline & & & $\begin{array}{l}\text { Baseline } \\
\left(\mathrm{FW}^{*}\right)\end{array}$ & $\begin{array}{l}1^{\text {st }} \text { follow up }\left(F^{*}\right) \\
\text { NVD - by } 2^{\text {nd }} \text { week } \\
\text { LSCS - by } 6^{\text {th }} \text { week }\end{array}$ & \\
\hline NVD & 36 & $26.3 \pm 3.94$ & $4.5 \pm 1.71$ & $2.4 \pm 1.24$ & $\mathrm{P}<0.01$ \\
\hline LSCS & 32 & $26.6 \pm 3.65$ & $5.8 \pm 2.09$ & $2.9 \pm 1.85$ & $\mathrm{P}<0.01$ \\
\hline
\end{tabular}

*FW - finger width; LSCS -Lower segment cesarean section; NVD -Normal vaginal delivery

Table 2: Reduction of DRA among NVD and LSCS Primiparous women during second follow up.

\begin{tabular}{|c|c|c|c|c|c|}
\hline \multirow{2}{*}{$\begin{array}{l}\text { Type of } \\
\text { delivery }\end{array}$} & \multirow{2}{*}{$\begin{array}{l}\text { No. of } \\
\text { participants }\end{array}$} & \multirow{2}{*}{$\begin{array}{l}\text { Mean age } \\
\text { (years) }\end{array}$} & \multicolumn{2}{|c|}{ Mean and SD of DRA } & \multirow[t]{2}{*}{ Paired t test } \\
\hline & & & $\begin{array}{l}\text { Baseline } \\
\left(\text { FW }^{*}\right)\end{array}$ & $\begin{array}{l}1^{\text {st }} \text { follow up }\left(\mathrm{FW}^{*}\right) \\
\text { NVD }- \text { by } 2^{\text {nd }} \text { week } \\
\text { LSCS - by } 6^{\text {th }} \text { week }\end{array}$ & \\
\hline NVD & 12 & $27.2 \pm 4.17$ & $4.5 \pm 1.78$ & $0.75 \pm 0.96$ & $\mathrm{P}<0.01$ \\
\hline LSCS & 9 & $26.2 \pm 2.93$ & $6.2 \pm 1.92$ & $1.3 \pm 0.67$ & $\mathrm{P}<0.01$ \\
\hline
\end{tabular}

*FW - finger width; LSCS -Lower segment cesarean section; NVD -Normal vaginal delivery

Table 3: Comparison of DRA change score between NVD and LSCS.

\begin{tabular}{|lllcc|}
\hline Type of delivery & No. of participants & Mean and SD of DRA & Independent $t$ value & Significance \\
\hline NVD & 12 & $3.8 \pm 1.46$ & -1.587 & $P=0.129 * *$ \\
\hline LSCS & 9 & $4.9 \pm 1.74$ & & \\
\hline *FW - finger width; LSCS -Lower segment cesarean section; NVD -Normal vaginal delivery; ** - Not significant
\end{tabular}

\section{Analysis of primiparous women with vaginal delivery}

Among 54 primiparous women with vaginal delivery, only 36 women $(26.3 \pm 3.94$ years $)$ regularly continued their exercise programme and revisited on the $2^{\text {nd }}$ week following delivery for reassessment of DRA. Eighteen women did not continue their exercise programme due to lack of time and were excluded. The mean difference 
between the pre- and post-intervention DRA $(\mathrm{n}=36)$ values was $2.1 \pm 1.16$ finger widths, which was statistically significant $(p<0.01$ [Table 1]) and also showed a large effect size $(\mathrm{d}=1.2)$.

\section{Analysis of Primiparous women with cesarean section}

Among 42 primiparous women with LSCS, only 32 women $(26.6 \pm 3.65$ years) continued their exercise programme regularly and revisited for further follow up at their $6^{\text {th }}$ post-operative week. The mean difference between the pre- and post-interventional value $(n=32)$ was $2.81 \pm 1.8$ finger widths, which was found to be statistically significant $(\mathrm{p}<0.01$ [Table 1]) with an effect size of $d=1.3$ regularly continued their exercise programme and revisited on the $2^{\text {nd }}$ week following delivery and they were reassessed for the DRA.

Primiparous women who had VD $(\mathrm{n}=12)$ and LSCS $(\mathrm{n}=$ 9) performed the exercise programme for the prescribed duration and revisited for their final follow up at their $6^{\text {th }}$ week and $10^{\text {th }}$ week following delivery respectively. Paired " $t$ " test values for both the groups showed significant difference in the size of DRA $(\mathrm{p}<0.01$ [Table 2]).

\section{Comparison of primiparous women with VD and LSCS}

The change score values of DRA from baseline to $2^{\text {nd }}$ follow up between VD and LSCS were analyzed using independent " $t$ " test. The results revealed that there was no significant difference between the groups ( $p>0.05$ [Table 3]) which indicated that both the groups showed the same level of improvement.

\section{DISCUSSION}

The present study investigated the effect of DRA corrective exercises in reducing the size of DRA in primiparous women. DRA corrective exercises performed from $4^{\text {th }}$ postnatal day by the primiparous women who had normal delivery and immediately after the $4^{\text {th }}$ week from the date of delivery by the primiparous women with cesarean section showed reduction in the size of DRA when they were followed up during their $6^{\text {th }}$ week and $10^{\text {th }}$ week respectively. Our study employed similar exercise protocol recommended by Hsia and Jones where the postnatal abdominal exercises were instituted after 48 hours. They have stated that the removal of stretch on the anterior abdominal wall immediately after delivery generally decreases the inter-recti distance.

There might be many reasons for the significant reduction in DRA observed in the current study. Firstly, the study incorporated TA training, to reduce DRA. "Bilateral activation of the TA can stabilize the ribs, linea alba and thoracolumbar fascia. When the TA is engaged, this shortens the rectus abdominis muscles and brings together the gap at the lineaalba. ${ }^{11}$ Moreover, cocontraction of pelvic floor and transverse abdominis is also emphazised in this study, as core strengthening exercises for the postpartum client should focus on the working relationship of the abdominal, back, diaphragm and pelvic floor muscles for the first 6 weeks postpartum. ${ }^{14}$

Performing sit-ups/diagonal curl ups without abdominal bracing with a weak transverse abdominis impairs its ability to "corset" and stabilize the abdomen, possibly exacerbating the diastasis recti ${ }^{14}$. Hence strengthening exercises for rectus abdominis through curl up exercises without bracing and for the oblique muscles through diagonal curl up exercises have been started once the DRA is reduced to 2 finger width without any bulge in the midline.

Secondly, the study has also incorporated approximation exercise which is an abdominal bracing exercise. This exercise emphasizes rectus abdominis muscle and minimizes the obliques. Acharry and Kutty found that abdominal exercises with bracing have been very effective in reducing DRA in early post-partum period. This indicates that in early post natal period, exercises seem to very effective, so this can be a nonsurgical solution for the Diastasis Recti Abdominis muscle.

In the current study, the participants with VD and LSCS followed the diastasis recti corrective exercises (TA strengthening, approximation exercises and pelvic floor exercises) as a 6 week protocol without any abdominal belt. Though the exercise programme was started after a month for the primiparous women with LSCS, both the groups (VD and LSCS) showed a good reduction in DRA. There is evidence for the 6 week protocol by ElMekawy et al., who has reported that postpartum women performing abdominal exercises for 6 weeks from the second day following vaginal delivery showed better improvement in abdominal muscle strength and a greater decrease in inter-recti distance (IRD) when compared to the women who used post-natal abdominal supporting belt. $^{15}$

This study has included primiparous women, as correction of DRA following first delivery through TA strengthening helps in preventing further separation of Rectus abdominis muscle in the next pregnancy. Moreover, few studies state that DRA above the umbilicus has similar prevalence in primiparae and multiparae and irrespective of parity, the distance between Rectus Abdominis Muscle (RAM) and the prevalence of Separation of Rectus Abdominis Muscle (SRAM) were greater in the supra-umbilical region and correlated with the age of the mother, BMI and infra-umbilical SRAM. ${ }^{6,17}$

The study had some limitations. The exercise sessions performed at home could not be supervised, and the participants were only motivated to perform the exercises by explaining the complications of DRA. However, an exercise diary filled by the participants was used to verify 
their compliance with the exercise programme. Future clinical trials with more samples with long-term follow up including a control group may help to further delineate whether resolution of DRA occur because of DRA corrective exercises or by chance. Palpation and calipers are used most often used to measure the IRD. Though ultrasonography is highly sensitive to changes in IRD, and is recommended as a valid measurement tool, this could not be used because therapists were not trained in using ultrasonography. However, Mota et al. showed that palpation of DRA width has sufficient reliability to be used in clinical practice. ${ }^{16}$ Hence, finger width measurement was used as an outcome measure in this study. The sample size of this study is very less. This study has not correlated Body mass index of the pregnant women and weight of the new born with the DRA only at the level of umbilicus and has included only primiparous women. Further studies could be done by comparing the size of DRA between the primiparous and multiparous women.

\section{CONCLUSION}

DRA corrective exercises performed by the primiparous women with DRA in their postpartum period immediately following ND or a month after the LSCS for 6 weeks were found to be effective in reducing DRA. The postnatal mothers need to be made aware of the importance of DRA corrective exercises in preventing further complications like altered posture, umbilical hernia and low back pain.

\section{ACKNOWLEDGEMENTS}

The authors kindly acknowledge and thank Dr. Lalitha and Dr. Ashokan Arumugam for their review of this paper and providing their valuable suggestions.

\section{Funding: No funding sources}

Conflict of interest: None declared

Ethical approval: The study was approved by the Institutional Ethics Committee of PSG Institute of Medical Sciences and Research, Coimbatore

\section{REFERENCES}

1. The Apple cart: a core concepts musculoskeletal group newsletter, 2010.

2. Parkeret MA. Diastasis Rectus Abdominis and Lumbo-Pelvic Pain and Dysfunction-Are They Related? Journal of Women's Health Physical Therapy. 2008;32:1.

3. Kutty RK. Abdominal Exercise with Bracing, A Therapeutic Efficacy in Reducing Diastasis-Recti
Among Postpartal Females. Int J Physiother Res. 2015;3(2):999-05.

4. Aguirre DA. Abdominal wall hernias: Imaging features, complication, and diagnostic pitfalls at multi-detector row CT. RSNA Education Exhibits. 2005;25(6).

5. Clarke V, Barton S. The 37-year-old primiparous woman: A challenge to the women's health physiotherapist? International organization of physical therapists in women's health. 2005.

6. Rett MT. Prevalence of diastasis of rectus abdominis immediately postpartum: Comparision between primiparae and multiparae. Rev Bras Fisioter. 2009;13(4):275-80.

7. Hsia M, Jones S. Natural resolution of rectus abdominis diastasis. Two single case studies. Australian Journal of Physiotherap. 2000;46:301-7.

8. Coldron Y, Stokes MJ. Postpartum characteristics of rectus abdominis on ultrasound imaging. Manual Therapy. 2008;13:112-21.

9. Spitznagleet TM. Prevalence of diastasis recti abdominis in a urogynecological patient population, International Urogynecology Journal. 2007;18(3).

10. Will AP. Pregnancy and Postpartum: A Guide for Singers. A Research Paper, Arizona State University, 2013.

11. Perry SE. Maternal Child Nursing Care. $5^{\text {th }}$ Edition, Elsevier Health Sciences, 2014.

12. Murray SS. Emily Slone McKinney, "Study Guide for Foundations of Maternal -Newborn and Women's Health Nursing. 6th edition, 2013, Elsevier Saunders.

13. Sullivan GM. Using effect size - or why the $P$ value is not enough. J Grad Med Educ. 2012;4(3):279-82.

14. IDEA Health and Fitness, "Inspire Women to Fitness: IDEA Health and Fitness Association, 2003.

15. El-Mekawy HS. Effect of abdominal exercises versus abdominal supporting belt on post-partum abdominal efficiency and rectus separation. International Scholarly and Scientific Research and Innovation. 2013;7(1).

16. Pascoal AG, Dionisio S. Inter-rectus distance in postpartum women can be reduced by isometric contraction of the abdominal muscles: a preliminary case-control study. Physiotherapy. 2014;100:344-8.

17. Rett MT, Almeida TV. Factors relating to mother and child associated with separation of the rectus abdominis muscle in immediate puerperium. Rev Bras Saúde Matern Infant Recife. 2014;14(1):73-80.

18. Candido G, Lo T, Janssen PA. Risk factors for diastasis of the recti abdominis. Journal of the Association of Chartered Physiotherapists in Women's Health, Autumn. 2005;97:49-54.

Cite this article as: Mahalakshmi V, Sumathi G, Chitra TV, Ramamoorthy V. Effect of exercise on diastasis recti abdominis among the primiparous women: a quasi-experimental study. Int J Reprod Contracept Obstet Gynecol 2016;5:4441-6. 